\title{
Clinical Validation of Kinematic Assessments of Post-Stroke Upper Limb Movements with a Multi- Joint Arm Exoskeleton
}

Florian Grimm ( $\square$ florian.grimm@med.uni-tuebingen.de )

Eberhard-Karls-Universitat Tubingen Medizinische Fakultat https://orcid.org/0000-0001-8862-814X Jelena Kraugmann

Eberhard Karls Universitat Tubingen

Georgios Naros

Eberhard Karls Universitat Tubingen

Alireza Gharabaghi

Eberhard Karls Universitat Tubingen

\section{Research}

Keywords: Human-machine interface, exoskeleton, sensorimotor interaction, virtual reality, hand-arm model, movement analysis, rehabilitation robotics, neurorehabilitation, stroke

Posted Date: August 7th, 2020

DOl: https://doi.org/10.21203/rs.3.rs-52210/v1

License: (9) This work is licensed under a Creative Commons Attribution 4.0 International License. Read Full License 


\section{Abstract}

Background: Robotic and gravity-balancing exoskeletons, originally designed for the rehabilitation training of neurological patients, are now being increasingly applied in objective and fine-grained sensorbased assessments of upper limb function. However, gravity compensation, inertia and damping properties of the exoskeleton interfere with the natural sensorimotor interaction, proprioceptive and visual feedback during movement execution. This may endanger the validity of the kinematic assessments in relation to the clinical outcome measures that they were supposed to reflect. Here, we applied

Methods: In a proof of concept study involving nineteen severely impaired chronic stroke patients, we assessed sensor-based kinematic data acquired with a multi-joint arm exoskeleton and compared it to the clinical outcome measure Upper Extremity Fugl-Meyer Assessment (UE-FMA) scale. During this assessment, real-time movement feedback of the system's seven degrees of freedom was provided with a biomorphic 3D virtual representation of the upper limb, including the proximal component of the arm. To align posture and to minimize the exoskeleton-patient interaction, the same position (neutral zero) with a distance of 90 degrees between forearm and upper arm was taken as the starting position for all assessments. Within self-contained tasks, we assessed separately and subsequently the range of motion/spatial posture of four single joints (i.e., joint angles of wrist, elbow, arm, and shoulder movement) and the closing and opening of the hand with a pressure sensor placed in the handle.

Results: A strong correlation was observed between wrist and elbow movements within the kinematic parameters $(r>0.7, p<0.003$; Bonferroni corrected). A multiple regression model predicted the UE-FMA significantly $\left(F(5,13)=12.22, p<0.0005\right.$, adj. $\left.R^{2}=0.83\right)$. Both shoulder rotation and grip pressure added significantly $(p<0.05)$ to the prediction with the standardized coefficients $\beta$ of 0.55 and 0.38 , respectively.

Conclusions: Exoskeleton-based evaluation of single-joint movements and grip force facilitates the assessment of upper limb kinematics after stroke with high structural and convergent validity. Proximal and distal measures may contribute independently to the prediction of the clinical status.

\section{Background}

The assessment of upper limb movements is crucial in monitoring and understanding sensorimotor recovery after stroke; kinematic assessments should enable a sensitive quantification of movement quality and distinguish between restitution and compensation in the weeks and months following the cerebrovascular event [1]. An increase in the assessment frequency by means of kinematic parameters could, therefore, optimize clinical assessment procedures and enhance the effectiveness of rehabilitation treatments [2].

In this context, three reviews have already summarized the state-of-the-art of upper limb kinematic parameters and how they correlate to clinical outcome measures following stroke [1-3]: 
Nordin and colleagues [3] reported that kinematic parameters describing movement accuracy were usually applied in chronic patients and correlated strongly with clinical assessments; parameters describing feed-forward sensorimotor control such as response latency, initiation time or initial speed were most often reported in studies of sub-acute patients and showed no correlation with clinical assessments. They also reported a lack of measurements for both coordinated movements and the proximal component of the upper limb. The challenge for robotic rehabilitation devices assessing the movement quality of stroke patients was also observed to lie in its ability to counterbalance robot dynamics and gravitational loading while also maintaining posture alignment during assessment sessions.

Of the forty-nine parameters identified by Do Tran and colleagues [2], movement speed, movement accuracy, peak speed, amount of speed peaks, movement distance and duration were the most frequently used. Thirteen studies investigated the correlation between instrumental parameters and clinical outcome measures. Although a number of parameters showed a significant association (correlation coefficient of more than 0.7 ), the majority were either weak (less than 0.3 ) or moderate $(0.3-0.7)$. When classified based on the International Classification of Functioning, Disability and Health (ICF) domains [4], the majority of the instrumental parameters were categorized into Body Functions and Structure, i.e. they addressed the impairment level that is most frequently clinically estimated by the Upper-Extremity FuglMeyer Assessment (UE-FMA) scale [5].

Schwarz and colleagues [1] differentiated kinematic upper limb assessments post-stroke with respect to the assessment task, measurement system and performance metrics. These were then evaluated on the basis of their clinimetric properties. This review identified a total of 151 different kinematic metrics that could be allocated to five task and three measurement system groups; the metrics task/movement time, number of movement onsets, number of movement ends, path length ratio, peak velocity, number of velocity peaks, trunk displacement, and shoulder flexion/extension all received an adequate evaluation for one clinimetric property. Twenty-two studies that investigated the correlation with clinical outcome measures (convergent validity) were identified. In exoskeletons, which are usually applied in severely impaired patients, the high interaction forces between the measurement system and patient due to friction, inertia, and arm weight support were particularly worthy of consideration. Furthermore, Schwarz and colleagues [1] proposed that the selected assessment task should be self-contained and that tasks trained during therapy should be avoided since these would confound the results for upper limb function by including task-specific learning effects [6].

In the present study, we aimed to enlarge upon some of the previous suggestions, e.g., to assess the proximal component of the upper limb and align posture also during the assessment, apply selfcontained assessment tasks, and minimize the exoskeleton-patient interaction to achieve high convergent validity of the applied kinematic parameters. We hypothesized that these goals could be achieved by a novel, self-contained and exoskeleton-based assessment protocol with biomorphic virtual reality feedback. 


\section{Method}

We recruited 19 stroke patients (8 females, mean age: $56 \pm 11$ [from 34 to 71] years) in the chronic phase after stroke (78 \pm 55 [from 8 to 244] months) who presented with severe and persistent hemiparesis (13 right-sided, 6 left-sided; 11 ischemic, 8 hemorrhagic) and who provided written informed consent (for demographic information see Table 1). The Upper Extremity Fugl-Meyer Assessment (UE-FMA) captured the motor function and contained the subscores A (upper extremity), B (wrist), C (hand) and D (coordination/speed), resulting in a total of max. 66 points. This clinical evaluation was performed by two examiners at the same time to minimize assessment variability. Clinical and kinematic assessments were done subsequently. The average UE-FMA score of the whole patient group was $16.1 \pm 5.2$ points; the individual patient scores had a range from 7 to 29 points (of 66 points); thus, the study included only severely impaired patients. This study was approved by the ethical review committee of the local medical faculty. 
Table 1

Demographic information.

\begin{tabular}{|c|c|c|c|c|c|c|}
\hline Patient No & Age & Type of Stroke & Gender & Side of stroke & Month post-stroke & UE-FMA \\
\hline 1 & 56 & hemorrhagic & male & right & 56 & 29 \\
\hline 2 & 52 & ischemic & male & right & 156 & 22 \\
\hline 3 & 68 & hemorrhagic & male & right & 34 & 16 \\
\hline 4 & 55 & hemorrhagic & male & right & 88 & 10 \\
\hline 5 & 67 & hemorrhagic & male & right & 75 & 7 \\
\hline 6 & 69 & ischemic & female & right & 130 & 16 \\
\hline 7 & 69 & ischemic & male & right & 81 & 14 \\
\hline 8 & 34 & hemorrhagic & male & right & 45 & 13 \\
\hline 9 & 63 & ischemic & female & right & 58 & 16 \\
\hline 10 & 59 & ischemic & female & left & 20 & 19 \\
\hline 11 & 63 & ischemic & female & left & 133 & 13 \\
\hline 12 & 51 & ischemic & female & right & 21 & 22 \\
\hline 13 & 56 & ischemic & female & right & 87 & 22 \\
\hline 14 & 49 & hemorrhagic & male & left & 69 & 21 \\
\hline 15 & 71 & hemorrhagic & male & right & 244 & 14 \\
\hline 16 & 41 & hemorrhagic & male & right & 62 & 9 \\
\hline 17 & 48 & ischemic & male & left & 8 & 13 \\
\hline 18 & 36 & ischemic & female & left & 32 & 16 \\
\hline 19 & 49 & ischemic & female & left & 81 & 14 \\
\hline
\end{tabular}

\section{Exoskeleton and 3D-Visualization:}

The basic methodology of our exoskeleton-based training and assessment setup has already been described in detail in previous studies and is cited here accordingly [7-9]: We used a commercially available (Armeo Spring, Hocoma, Volketswil, Switzerland) rehabilitation exoskeleton for shoulder (arm rotation, arm elevation), elbow (flexion/extension) and wrist joints (flexion/extension) with seven degrees of freedom to provide gravity-balancing support for the paretic arm and simultaneous registration of movement kinematics and grip force.

This device enabled us to make individual adjustments of gravity compensation, thereby supporting patients with severe impairment in performing task-oriented practice within a motivating virtual 
environment. To align posture and to minimize the exoskeleton-patient interaction, the same position (neutral zero) with a distance of 90 degrees between forearm and upper arm, with the shoulder being adducted to the trunk and with the thumb pointing upwards, was applied as the starting position for all assessments. In accordance with the manufacturer's instructions, the length of the different components of the exoskeleton with regard to the wrist, forearm and upper arm were adjusted to suit the individual anatomical proportions of each patient. Gravity compensation was set according to the manufacturer's instruction, thereby, allowing for a complete gravity compensation of the upper limb in the neutral zero position. In this context, a better understanding of the weight compensation provided by this device may help to fully utilize it in clinical and research settings [10]. A file mapping communication protocol was used to read the real-time movement data, as originally represented in the angles of all arm joints, and the grip force measured by the device from a shared memory block.

Using the real-time sensor data of the exoskeleton to display a three-dimensional multi-joint visualization of the user's arm in virtual reality (VR), we extended these features in-house to provide both visual and auditory instructions and feedback for the patient. Since our exoskeleton-based rehabilitation interventions were already using this VR set-up, we applied the same technology also for the assessment protocol to avoid a methodological disruption of the integrated training and assessment sessions. The aim of this VR approach was, furthermore, to standardize the evaluation independent of the interaction of an examiner to reduce assessment variability. The system's features allow for further optimization (e.g., multimodal feedback, personalized content, gamification) in future. The real-time sensor data enabled us to display a natural virtual 3D representation of the patient's arm on a computer screen. This provides the patient with additional visual feedback on how the movement was performed. The virtual arm engine was programmed in a Microsoft XNA framework. The arm model utilized by the engine was constructed as a meshed bone-skin combination with 56 bones which were modelled as interconnected bodies in the simulation. (3Ds Max 2010TM, Autodesk). This model included 14 finger bones, 11 hand route bones and one bone for each shoulder, forearm and upper arm for each side of the body [7]. The real-time sensor data modulated the 3D model. Specifically, the joint angles and grip forces of the device measured with the exoskeleton were used to modify the bone-vectors (i.e., position of the bone objects in CAD space) of the meshed model in accordance with the movements of the user, thereby providing online closed-loop feedback. The joint angles of the exoskeleton were directly represented in virtual reality, while the grip forces were amplified to feedback natural hand function.

\section{Movement assessment design:}

The positioning of the patient in the exoskeleton, including the complete movement assessment $(\sim 10$ $\mathrm{min})$ and the clinical evaluation with the UE-FMA score ( $30 \mathrm{~min})$ were performed on the same day. To facilitate an efficient evaluation of the motor abilities of severely affected stroke patients, a kinematic registration of the arm was conducted in one self-contained task session.

A software design instructed the patients by 3D-arrows, text and tone messages to repeat single-joint movements while providing feedback related to the performed movements and the range of motion 
(Figure 1). The simple instructions and single-joint movements ensured that the self-contained movements could be performed by patients of all cognitive levels. Since these tasks were designed to measure the maximum range of motion of single-joints, reference points did not require tracking and so no overshoots - such as may be observed during 3D motion tracking when 3D rendering is displayed on a 2D [11] - were found to occur. However, previous work of our group using a different evaluation program for activities of daily living (ADL) captured movement quality, i.e., precisely such overshoots, and showed that patients were inaccurate in comparison to healthy subjects. These inaccuracies decreased in the course of a four-week training program $[7,9]$. In this study, we designed simple, self-contained tasks that minimize patient-exoskeleton interactions and do not rely on learning [1], thus preventing potential confounds that are related to human-device interactions but not to motor recovery [6].

In order to develop a fast and practical assessment for severely impaired stroke patients that could be applied in the context of daily rehabilitation sessions, the overall number of evaluated parameters was restricted. The single-joint movements of this task were, moreover, carefully selected to be independent of the exoskeleton environment, i.e., they could also be translated to the environment outside the exoskeleton if the patients' impairments still enabled them to perform these without gravitycompensation. The following joints were measured subsequently and selectively: grip pressure (difference between closing and opening the hand), wrist movement (flexion and extension), elbow movement (flexion and extension), upper arm elevation and shoulder rotation; shoulder ab- and adduction was limited due to the physical constraints of the exoskeleton. Each movement was performed 5 times, allowing movement for $5 \mathrm{~s}$ in each direction followed by a rest period of $5 \mathrm{~s}$.

Our patients were so severely impaired that the range of motion of the exoskeleton was not exceeded. Therefore, no saturation occurred in this study. Previous studies, in which activities of daily living with this exoskeleton were performed with healthy subjects, also showed that the range of motion was not limited $[7,9]$.

All joint movement data for the wrist, elbow, upper arm, and shoulder were recorded during the exercises in ${ }^{\circ}$. The grip pressure was estimated in kilopascal $(\mathrm{kPa})$.

\section{Statistics and data evaluation}

Statistical analysis was performed on a Matlab 2010b Engine and SPSS (IBM SPSS Statistics for Windows, Version 22.0. Armonk, NY: IBM Corp.). The extent of the kinematic parameters was calculated as a mean over the trials.

A multiple regression was performed to predict the UE-FMA score from grip pressure, wrist movement, elbow movement, arm elevation, and shoulder rotation. The linearity was assessed by partial regression plots and a plot of studentized residuals against the predicted values. The independence of residuals was assessed by a Durbin-Watson statistic. The assumption of normality was assessed by a Q-Q Plot. The significance level was set at $p=0.05$ for all tests. 
A Pearson's product-moment correlation was estimated to assess the relationship within and between the subscores A-D of the UE-FMA and the kinematic parameters. The analyses showed a linear relationship with the variables being normally distributed as assessed by the Shapiro-Wilk test $(p>.05)$; there were no outliers.

\section{Results}

Patients became easily accustomed to the assessment setup without adverse effects. The system and the training-software ran smoothly throughout the evaluation. Due to the gravity compensation of the exoskeleton, all severely impaired patients were able to perform the movement assessment task.

\section{Kinematic parameters}

On average, 6 minutes and 15 seconds were required to register the patients' movement abilities. One operator (who positioned the patients in the exoskeleton) was present during the assessment but did not need to intervene in the evaluation procedure since the instructions, feedback and exercises ran smoothly. The ensuing kinematic performance parameters are presented in Figure 2, and their correlations with the UE-FMA score in Figure 3. Exemplary real-time kinematic data of three patients is illustrated in Figure 4.

Within the kinematic parameters, there was a strong correlation between wrist and elbow movements $(r>$ 0.7, p<0.003; Bonferroni corrected, Table 2), thereby representing the known flexor hypertonia (spasticity) of the wrist and elbow in these severely affected stroke patients (structural validity). Between the kinematic and clinical parameters, there was a moderate to high correlation between all instrumental measures and the UE-FMA subscores A (upper extremity) and D (coordination/speed).

A multiple regression analysis was performed to predict the UE-FMA score from grip pressure, wrist movement, elbow movement, arm elevation, and shoulder rotation. Linearity was assessed by partial regression plots and a plot of studentized residuals against the predicted values. Independence of residuals was assessed by a Durbin-Watson statistic of 1.97 .

Homoscedasticity was assessed by visual inspection of a plot of studentized residuals versus unstandardized predicted values. Since none of the tolerance values exceeded 0.1 , there was no evidence of multicollinearity. No studentized deleted residuals were found to be greater than \pm 3 standard deviations, no leverage values were greater than 0.2 , and the values for Cook's distance were above 1 . As assessed by a Q-Q Plot, the assumption of normality was confirmed.

A multiple regression model predicted the UE-FMA significantly $\left(F(5,13)=12.22, p<0.0005\right.$, adj. $R^{2}=$ 0.83 ). Shoulder rotation and grip pressure, with standardized coefficients $\beta$ of 0.55 and 0.38 , respectively, both added significantly $(p<0.05)$ to the prediction (Table 3$)$. In the post hoc power analysis [12], the predicted multiple regression model $(p<0.001, d f=5$ and $n=19)$ had a statistical power between 0.83 and 0.89 . 
Table 2

Cross-correlation of kinematic parameters and correlations with the UE-FMA sub scores A-B (r, upper section) and corresponding $p$-values ( $p$, lower section).

*Strong correlation: $r>0.7, p<0.003$ (Bonferroni corrected)

\begin{tabular}{|c|c|c|c|c|c|c|}
\hline & & grip & wrist & elbow & arm & shoulder \\
\hline \multirow[t]{9}{*}{$\mathrm{r}$} & grip in $\mathrm{kPa}$ & 1.000 & .394 & .348 & .463 & .267 \\
\hline & wrist movement in ${ }^{\circ}$ & .394 & 1.000 & $.735^{\star}$ & .422 & .475 \\
\hline & elbow movement in ${ }^{\circ}$ & .348 & $.735^{\star}$ & 1.000 & .547 & .614 \\
\hline & arm elevation in ${ }^{\circ}$ & .463 & .422 & .547 & 1.000 & .543 \\
\hline & shoulder movement in ${ }^{\circ}$ & .267 & .475 & .614 & .543 & 1.000 \\
\hline & UE-FMA subscore A & $.624^{*}$ & $.618 *$ & $.790 *$ & $.725^{\star}$ & $.588 *$ \\
\hline & UE-FMA subscore B & .434 & .187 & .107 & 0.334 & .355 \\
\hline & UE-FMA subscore C & .120 & .043 & .120 & .119 & -.190 \\
\hline & UE-FMA subscore D & $.593^{*}$ & $.605^{\star}$ & $.718 *$ & $.585^{*}$ & .388 \\
\hline \multirow[t]{9}{*}{$p$} & grip in $\mathrm{kPa}$ & . & .048 & .072 & .023 & ,134 \\
\hline & wrist movement in ${ }^{\circ}$ & .048 & . & .000 & .036 & .020 \\
\hline & elbow movement in ${ }^{\circ}$ & .072 & .000 & . & .008 & .003 \\
\hline & arm elevation in ${ }^{\circ}$ & .023 & .036 & .008 & $\cdot$ & .008 \\
\hline & shoulder movement in ${ }^{\circ}$ & 134 & .020 & .003 & .008 & . \\
\hline & UE-FMA subscore A & .004 & .005 & ,000 & .000 & .008 \\
\hline & UE-FMA subscore B & .063 & .443 & .663 & .162 & .136 \\
\hline & UE-FMA subscore $\mathrm{C}$ & .625 & .860 & .626 & .627 & .435 \\
\hline & UE-FMA subscore D & .007 & .006 & .001 & .008 & .100 \\
\hline
\end{tabular}


Table 3

Summary of Multiple Regression Analysis. ${ }^{*} \mathrm{p}<$

$0.05, \mathrm{~B}=$ unstandardized regression coefficient;

SEB $=$ Standard error of the coefficient; $\beta=$ standardized coefficient.

\begin{tabular}{|llll|}
\hline Variable & B & SEB & $\boldsymbol{\beta}$ \\
\hline Intercept & 4.341 & 1.670 & \\
\hline grip pressure & 0.253 & 0.90 & $\mathbf{0 . 3 8 0 ^ { * }}$ \\
\hline wrist movement & -0.009 & 0.056 & -0.027 \\
\hline elbow movement & 0.049 & 0.064 & 0.152 \\
\hline arm elevation & 0.039 & 0.054 & 0.111 \\
\hline shoulder rotation & 0.462 & 0.130 & $\mathbf{0 . 5 5 2 *}$ \\
\hline
\end{tabular}

\section{Discussion}

In this proof of concept study, we used an exoskeleton-based assessment protocol to demonstrate the convergent validity of the acquired kinematic data in comparison with the UE-FMA clinical outcome measure in severely impaired stroke patients. The characteristics of the assessment protocol were as follows:

1. It provides real-time movement feedback of the measurement system's seven degrees of freedom by virtue of a biomorphic virtual representation of the upper limb, including the proximal component of the upper limb.

2. It adjusts the device position and length of the forearm, upper arm and wrist appliances to the individual anatomical proportions to align posture and reduce patient movement within the exoskeleton.

3. It applies the same position (neutral zero) with a distance of 90 degrees between forearm and upper arm as the starting position for all assessments to minimize the exoskeleton-patient interaction.

4. It assesses, separately and subsequently as self-contained tasks, the range of motion/spatial posture of single joints (i.e., joints' angles) and the closing and opening of the hand with a pressure sensor placed in the handle.

The majority of stroke patients will presumably never regain full function of the affected limb $[13,14]$. The impairment of an upper extremity is a decisive factor for their diminished quality of life [15]. Early and high-dose movement therapies are therefore relevant for clinically meaningful improvements [16]. Furthermore, an assessment of upper limb movements is crucial in monitoring and understanding sensorimotor recovery [17]. 
Assistive technologies supporting robot-assisted and gravity-balancing training are therefore now under investigation as a means of increasing and standardizing the scope of therapy and assessment for stroke rehabilitation. These devices facilitate intensive, high-dose and repetitive exercises of activities of daily living ( $A D L)$, and may also support the therapists by an objective assessment of the upper limb. In this context, the gravity-balancing support by a seven degree-of-freedom arm exoskeleton may be particularly useful during the rehabilitation training and assessment of severely affected stroke patients $[18,19]$. Together with other assessment options, this arm exoskeleton, a version of which is now commercially available, is suitable for real-time recording of wrist/arm/shoulder movements and finger strength.

In this context, extended custom-made soft- and hardware applications have been implemented and investigated to augment the functionalities: Online feedback of extent of movement and quality for the assisted ADL-like exercises [7, 8], closed-loop task difficulty adaptation of these virtual reach-to-grasp tasks [9], and hybrid exoskeletons including adaptive neuromuscular stimulation [20], additional brain control [21] and robotic support with active actuation [22].

It is, however, important to determine the convergent validity of these exoskeleton-based assessments, i.e., the degree to which the correlation of metrics to clinical scales is consistent with the hypothesis [1]. The Fugl-Meyer assessment [5] is still the gold standard and is commonly used for clinical movement assessment in hemiparetic stroke patients. Excellent interrater and intrarater reliability have been demonstrated, and the score is responsive to changes in motor impairment [23]. This assessment is, however, known to have its limitations. One study suggests that the sensory scale's psychometric properties should not be used to assess chronic stroke patients [24]. The pain domain could be a confounding variable to the joint range of motion, which would render it unnecessary. Moreover, distal fine motor functions may be underrepresented. In addition, a ceiling effect of the motor function has been reported [25]. In this context, provided that they show convergent validity with the UE-FMA, the use of exoskeleton-based measurements may allow for a finer and more specific registration of movements [3].

Furthermore, the UE-FMA is relatively time-consuming and cannot be performed in the context of an exercise session, e.g., to track the improvement during a training period. With the assessment task presented here, detailed information about the progress on the impairment level can be provided during each training unit with minimal additional time expenditure. Importantly, this information is not confounded, as the task is self-contained and specified for the evaluation of motor impairment [1]. In particular, this assessment task does not include those training tasks applied during exoskeleton-based therapy, and the results pertaining to upper limb function are, therefore, not confounded by task-specific learning effects [6].

Our setup contained an integrated virtual reality module to provide immediate and continuous feedback of the movement extent. Such approaches are vital to motor learning in rehabilitation $[7,9,26]$ and have been expanded here to the area of instrumental assessment. In this context, virtual reality is often used to provide feedback and might be beneficial during rehabilitation $[8,27-29]$. To build an interactive interface 
for such purposes, movement data from the patients is acquired by various mechanical or optical systems, e.g., CyberGlove [30], orthotic exoskeletons [18], gaming systems [31-33], or in combination with robotic systems for haptic feedback such as Rutgers Master II-ND haptic glove, MIT-Manus [34] or ARMIN [35]. Devices such as the Armeo Spring, ARMIN [35], Pneu-Wrex [36], ULEX07 [37], have the advantage of providing at least partial kinematic registration of the upper extremity movement for different joints. By contrast, systems such as the MIT-Manus [34], ReaPLAN [38, 39], ReoGo [40], Planar robot [40], and PUPArm [41] allow for endpoint-based alignment. With these devices, the movement of the shoulder and upper arm is estimated as a surrogate parameter, and not directly via sensors. The algorithm proposed in this study can be easily transferred to those former systems that allowed the registration of the different joints. This approach would, therefore, provide a standardized assessment that could be performed on different devices and enable better comparisons of the studies.

We assessed all single-joint angles in a standardized fashion that is often applied in the clinical context of neurological and orthopedic evaluations (i.e., beginning from the normal zero position with 90 degrees distance between forearm and upper arm). This distinguishes our approach from previous methods for kinematic movement assessment that were acquired during specific exercises with more complex movements [42]. Furthermore, various biomechanical parameters are often used for classification such as inter-joint coordination [43, 44], temporal efficiency [45-47], movement speed [48-50], smoothness [51] or accuracy [52]. Such classification algorithms tend to be rather device-specific and are not easily transferable to other hard- and software settings. Furthermore, sometimes even elaborate models are necessary, using, for example, artificial neuronal networks, to generate clinically relevant parameters [53]. On the whole, a large number of the identified kinematic parameters were derived from existing exercises within individual systems and compared with the UE-FMA score. Range-of-motion-based evaluation systems, for example, are available for task-related assessments [54, 55]. However, only very little data is available concerning single-joint-based movement assessment of, for example, wrist motion [56] or elbow movement [57]; moreover, no significant results are available for these approaches. Only when shoulder rotation was included, were significant correlations with clinical scores reported [58, 59]; this was also the case when compensatory movement patterns, which contribute to pathological upper arm coordination, were investigated $[52,60]$.

The choice of task, measurement system and metrics for the presented upper limb kinematic assessment addressed first and foremost the impairment level of severely affected patients who were unable to perform these movements without gravity-balancing. We demonstrated that shoulder rotation, a frequently neglected proximal component of kinematic assessments of the upper limb [3], was the measure with the most relevant contribution to the prediction of the clinical outcome measure. This may be related to the characteristics of the applied clinical outcome measure for stroke patients, i.e., the UEFMA scale. The over-representation of the shoulder in the different evaluation tasks, a known feature of the UE-FMA, contributes to the overall score [25].

The second-best parameter that predicted the clinical status was, notably, the grip force, which tends to be under-represented in the UE-FMA. This finding may suggest that this fairly straightforward 
instrumental measure - which can be easily acquired even without an exoskeleton - may be best suited to a practical quantification of clinically relevant kinematics in a wide variety of patients after stroke. However, future work would need to study larger and independent sample sizes to confirm the predictive properties of these kinematic parameters. Furthermore, as there is no benchmark to compare the range of motion measured with the exoskeleton, in future work, participants may perform the task twice with two different operators to provide a measure of repeatability.

\section{Conclusion}

In conclusion, exoskeleton-based feedback of single-joint angles and grip force facilitates the rapid kinematic assessment of the range of motion of the upper limb after stroke with high structural and convergent validity. Important features of our approach were self-contained assessment tasks, minimized exoskeleton-patient interaction and biomorphic virtual reality feedback. Proximal and distal measures may contribute independently to the prediction of the clinical status.

\section{Declarations}

Acknowledgments This work was supported by the German Federal Ministry of Education and Research [BMBF, INERLINC 16SV8174]. AG was supported by grants from the Baden-Wuerttemberg Foundation and the German Federal Ministry of Education and Research. We also acknowledge support by the Open Access Publishing Fund of the University of Tuebingen. The authors report no conflict of interest.

\section{Ethics approval and consent to participate}

This study was approved by the ethics committee of the medical faculty of the University of Tuebingen. Written, informed consent was obtained before participation.

\section{Consent for publication}

Not applicable

\section{Availability of data and materials}

The datasets are available from the corresponding author on reasonable request.

\section{Competing interests}

The authors declare that the research was conducted in the absence of any commercial or financial relationships that could be construed as a potential conflict of interest.

\section{Funding}

This work was supported by the German Federal Ministry of Education and Research [BMBF, INERLINC 16SV8174]. AG was supported by grants from the Baden-Wuerttemberg Foundation and the German 
Federal Ministry of Education and Research. We also acknowledge support by the Open Access Publishing Fund of the University of Tuebingen. The authors report no conflict of interest. The funding body had no role in the design of the study and collection, analysis, interpretation of data and writing the manuscript.

\section{Authors' contributions}

Conceptualization: FG, AG; Methodology: FG; Programming of 3D applications: FG; Realization of the clinical study and patient care: FG, JK, GN; Statistical evaluation: FG, Writing - Original Draft Preparation: FG, AG; Writing - Review \& Editing: FG, JK, GN, AG; Visualization: FG, Supervision: FG, AG; Project Administration: FG, AG; Funding Acquisition: AG. All authors read and approved the final manuscript.

\section{References}

1. Schwarz, A., et al., Systematic Review on Kinematic Assessments of Upper Limb Movements After Stroke. Stroke, 2019. 50(3): p. 718-727.

2. Tran, V.D., P. Dario, and S. Mazzoleni, Kinematic measures for upper limb robot-assisted therapy following stroke and correlations with clinical outcome measures: A review. Med Eng Phys, 2018. 53: p. 13-31.

3. Nordin, N., S.Q. Xie, and B. Wunsche, Assessment of movement quality in robot-assisted upper limb rehabilitation after stroke: a review. J Neuroeng Rehabil, 2014. 11: p. 137.

4. Organization, W.H., Towards a common language for functioning, disability, and health: ICF. The international classification of functioning, disability and health, 2002.

5. Fugl-Meyer, A.R., et al., The post-stroke hemiplegic patient. 1. a method for evaluation of physical performance. Scand J Rehabil Med, 1975. 7(1): p. 13-31.

6. Schweighofer, N., et al., Dissociating motor learning from recovery in exoskeleton training post-stroke. J Neuroeng Rehabil, 2018. 15(1): p. 89.

7. Grimm, F., G. Naros, and A. Gharabaghi, Compensation or Restoration: Closed-Loop Feedback of Movement Quality for Assisted Reach-to-Grasp Exercises with a Multi-Joint Arm Exoskeleton. Front Neurosci, 2016. 10: p. 280.

8. Lewis, G.N. and J.A. Rosie, Virtual reality games for movement rehabilitation in neurological conditions: how do we meet the needs and expectations of the users? Disabil Rehabil, 2012. 34(22): p. 1880-6.

9. Grimm, F., G. Naros, and A. Gharabaghi, Closed-Loop Task Difficulty Adaptation during Virtual Reality Reach-to-Grasp Training Assisted with an Exoskeleton for Stroke Rehabilitation. Front Neurosci, 2016. 10: p. 518.

10. Perry, B.E., E.K. Evans, and D.S. Stokic, Weight compensation characteristics of Armeo(R)Spring exoskeleton: implications for clinical practice and research. J Neuroeng Rehabil, 2017. 14(1): p. 14. 
11. Gerig, N., et al., Missing depth cues in virtual reality limit performance and quality of three dimensional reaching movements. PLoS One, 2018. 13(1): p. e0189275.

12. Lenth, R.V., Statistical power calculations. J Anim Sci, 2007. 85(13 Suppl): p. E24-9.

13. Kwakkel, G., et al., Probability of regaining dexterity in the flaccid upper limb: impact of severity of paresis and time since onset in acute stroke. Stroke, 2003. 34(9): p. 2181-6.

14. Jorgensen, H.S., et al., Stroke. Neurologic and functional recovery the Copenhagen Stroke Study. Phys Med Rehabil Clin N Am, 1999. 10(4): p. 887-906.

15. Huang, Y.H., et al., Predictors of change in quality of life after distributed constraint-induced therapy in patients with chronic stroke. Neurorehabil Neural Repair, 2010. 24(6): p. 559-66.

16. Pollock, A., et al., Physical rehabilitation approaches for the recovery of function and mobility following stroke. Cochrane Database Syst Rev, 2014(4): p. CD001920.

17. Rudhe, C., et al., Reliability of movement workspace measurements in a passive arm orthosis used in spinal cord injury rehabilitation. J Neuroeng Rehabil, 2012. 9: p. 37.

18. Housman, S.J., K.M. Scott, and D.J. Reinkensmeyer, A randomized controlled trial of gravitysupported, computer-enhanced arm exercise for individuals with severe hemiparesis. Neurorehabil Neural Repair, 2009. 23(5): p. 505-14.

19. Bertani, R., et al., Effects of robot-assisted upper limb rehabilitation in stroke patients: a systematic review with meta-analysis. Neurol Sci, 2017. 38(9): p. 1561-1569.

20. Grimm, F. and A. Gharabaghi, Closed-Loop Neuroprosthesis for Reach-to-Grasp Assistance: Combining Adaptive Multi-channel Neuromuscular Stimulation with a Multi-joint Arm Exoskeleton. Front Neurosci, 2016. 10: p. 284.

21. Grimm, F., et al., Hybrid Neuroprosthesis for the Upper Limb: Combining Brain-Controlled Neuromuscular Stimulation with a Multi-Joint Arm Exoskeleton. Front Neurosci, 2016. 10: p. 367.

22. Brauchle, D., et al., Brain state-dependent robotic reaching movement with a multi-joint arm exoskeleton: combining brain-machine interfacing and robotic rehabilitation. Front Hum Neurosci, 2015. 9: p. 564.

23. Page, S.J., G.D. Fulk, and P. Boyne, Clinically important differences for the upper-extremity Fugl-Meyer Scale in people with minimal to moderate impairment due to chronic stroke. Phys Ther, 2012. 92(6): p. 791-8.

24. Lin, J.H., et al., Psychometric properties of the sensory scale of the Fugl-Meyer Assessment in stroke patients. Clin Rehabil, 2004. 18(4): p. 391-7.

25. Gladstone, D.J., C.J. Danells, and S.E. Black, The fugl-meyer assessment of motor recovery after stroke: a critical review of its measurement properties. Neurorehabil Neural Repair, 2002. 16(3): p. 232-40.

26. van Vliet, P.M. and G. Wulf, Extrinsic feedback for motor learning after stroke: what is the evidence? Disabil Rehabil, 2006. 28(13-14): p. 831-40.

27. Sveistrup, H., Motor rehabilitation using virtual reality. J Neuroeng Rehabil, 2004. 1(1): p. 10. 
28. Laver, K.E., et al., Virtual reality for stroke rehabilitation. Cochrane Database Syst Rev, 2017. 11: p. CD008349.

29. Cameirao, M.S., et al., Neurorehabilitation using the virtual reality based Rehabilitation Gaming System: methodology, design, psychometrics, usability and validation. J Neuroeng Rehabil, 2010. 7: p. 48.

30. Boian, R., et al., Virtual reality-based post-stroke hand rehabilitation. Stud Health Technol Inform, 2002. 85: p. 64-70.

31. Yavuzer, G., et al., "Playstation eyetoy games" improve upper extremity-related motor functioning in subacute stroke: a randomized controlled clinical trial. Eur J Phys Rehabil Med, 2008. 44(3): p. 23744.

32. Saposnik, G., et al., Effectiveness of virtual reality using Wii gaming technology in stroke rehabilitation: a pilot randomized clinical trial and proof of principle. Stroke, 2010. 41(7): p. 1477-84.

33. Yong Joo, L., et al., A feasibility study using interactive commercial off-the-shelf computer gaming in upper limb rehabilitation in patients after stroke. J Rehabil Med, 2010. 42(5): p. 437-41.

34. Krebs, H.I., et al., Robot-aided neurorehabilitation: from evidence-based to science-based rehabilitation. Top Stroke Rehabil, 2002. 8(4): p. 54-70.

35. Staubli, P., et al., Effects of intensive arm training with the rehabilitation robot ARMin II in chronic stroke patients: four single-cases. J Neuroeng Rehabil, 2009. 6: p. 46.

36. Reinkensmeyer, D.J., et al., Do robotic and non-robotic arm movement training drive motor recovery after stroke by a common neural mechanism? Experimental evidence and a computational model. Conf Proc IEEE Eng Med Biol Soc, 2009. 2009: p. 2439-41.

37. Kim, H., et al., Kinematic data analysis for post-stroke patients following bilateral versus unilateral rehabilitation with an upper limb wearable robotic system. IEEE Trans Neural Syst Rehabil Eng, 2013. 21(2): p. 153-64.

38. Gilliaux, M., et al., A robotic device as a sensitive quantitative tool to assess upper limb impairments in stroke patients: a preliminary prospective cohort study. J Rehabil Med, 2012. 44(3): p. 210-7.

39. Gilliaux, M., et al., Using the robotic device REAplan as a valid, reliable, and sensitive tool to quantify upper limb impairments in stroke patients. J Rehabil Med, 2014. 46(2): p. 117-25.

40. Kitago, T., et al., Robotic therapy for chronic stroke: general recovery of impairment or improved taskspecific skill? J Neurophysiol, 2015. 114(3): p. 1885-94.

41. Lledo, L.D., et al., A Comparative Analysis of 2D and 3D Tasks for Virtual Reality Therapies Based on Robotic-Assisted Neurorehabilitation for Post-stroke Patients. Front Aging Neurosci, 2016. 8: p. 205.

42. Veerbeek, J.M., et al., Effects of Robot-Assisted Therapy for the Upper Limb After Stroke. Neurorehabil Neural Repair, 2017. 31(2): p. 107-121.

43. Dukelow, S.P., et al., The independence of deficits in position sense and visually guided reaching following stroke. J Neuroeng Rehabil, 2012. 9: p. 72. 
44. Volpe, B.T., et al., Intensive sensorimotor arm training mediated by therapist or robot improves hemiparesis in patients with chronic stroke. Neurorehabil Neural Repair, 2008. 22(3): p. 305-10.

45. Zollo, L., et al., Quantitative evaluation of upper-limb motor control in robot-aided rehabilitation. Med Biol Eng Comput, 2011. 49(10): p. 1131-44.

46. Johnson, M.J., et al., Bilateral assessment of functional tasks for robot-assisted therapy applications. Med Biol Eng Comput, 2011. 49(10): p. 1157-71.

47. Coderre, A.M., et al., Assessment of upper-limb sensorimotor function of subacute stroke patients using visually guided reaching. Neurorehabil Neural Repair, 2010. 24(6): p. 528-41.

48. Mazzoleni, S., et al., Mechanisms of motor recovery in chronic and subacute stroke patients following a robot-aided training. IEEE Trans Haptics, 2014. 7(2): p. 175-80.

49. Longhi, M., et al., Instrumental indices for upper limb function assessment in stroke patients: a validation study. J Neuroeng Rehabil, 2016. 13(1): p. 52.

50. Laczko, J., et al., Inter-Joint Coordination Deficits Revealed in the Decomposition of Endpoint Jerk During Goal-Directed Arm Movement After Stroke. IEEE Trans Neural Syst Rehabil Eng, 2017. 25(7): p. 798-810.

51. Pila, O., et al., Pattern of improvement in upper limb pointing task kinematics after a 3-month training program with robotic assistance in stroke. J Neuroeng Rehabil, 2017. 14(1): p. 105.

52. Cirstea, M.C. and M.F. Levin, Compensatory strategies for reaching in stroke. Brain, 2000. 123 (Pt 5): p. $940-53$.

53. Krebs, H.I., et al., Robotic measurement of arm movements after stroke establishes biomarkers of motor recovery. Stroke, 2014. 45(1): p. 200-4.

54. Kahn, L.E., et al., Robot-assisted reaching exercise promotes arm movement recovery in chronic hemiparetic stroke: a randomized controlled pilot study. J Neuroeng Rehabil, 2006. 3: p. 12.

55. Iwamuro, B.T., et al., Effect of a gravity-compensating orthosis on reaching after stroke: evaluation of the Therapy Assistant WREX. Arch Phys Med Rehabil, 2008. 89(11): p. 2121-8.

56. Mazzoleni S, C.R., Posteraro F, Carroza MC, Effects of robot-assisted wrist therapy in chronic stroke patients: a kinematic approach. The Fourth IEEE RAS/EMBS International Conference on Biomedical Robotics and Biomechatronics. Rome, 2012(2012): p. 1978-1982.

57. Toth A, F.G., Arz G, Passive robotic movement therapy of the spastic hemiparetic arm with REHAROB: Report of the first clinical test and the follow-up system improvement. 2005 IEEE 9th International Conference on Rehabilitation Robotics. Chicago, IL, 2005. 2005: p. 127-130.

58. Fazekas, G., et al., Robot-mediated upper limb physiotherapy for patients with spastic hemiparesis: a preliminary study. J Rehabil Med, 2007. 39(7): p. 580-2.

59. Hogan, N. and D. Sternad, Sensitivity of smoothness measures to movement duration, amplitude, and arrests. J Mot Behav, 2009. 41(6): p. 529-34.

60. Cirstea, M.C. and M.F. Levin, Improvement of arm movement patterns and endpoint control depends on type of feedback during practice in stroke survivors. Neurorehabil Neural Repair, 2007. 21(5): p. 
398-411.

\section{Figures}

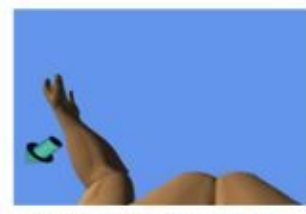

Shoulder rotation

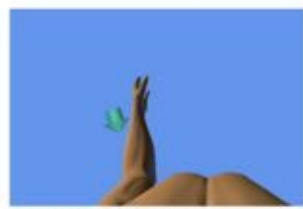

Arm elevation
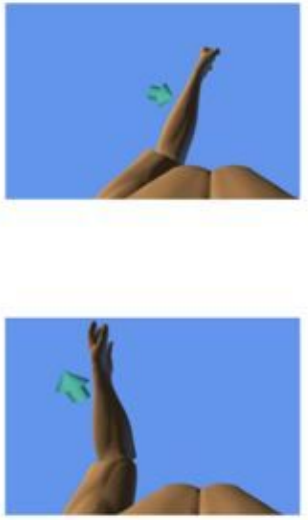
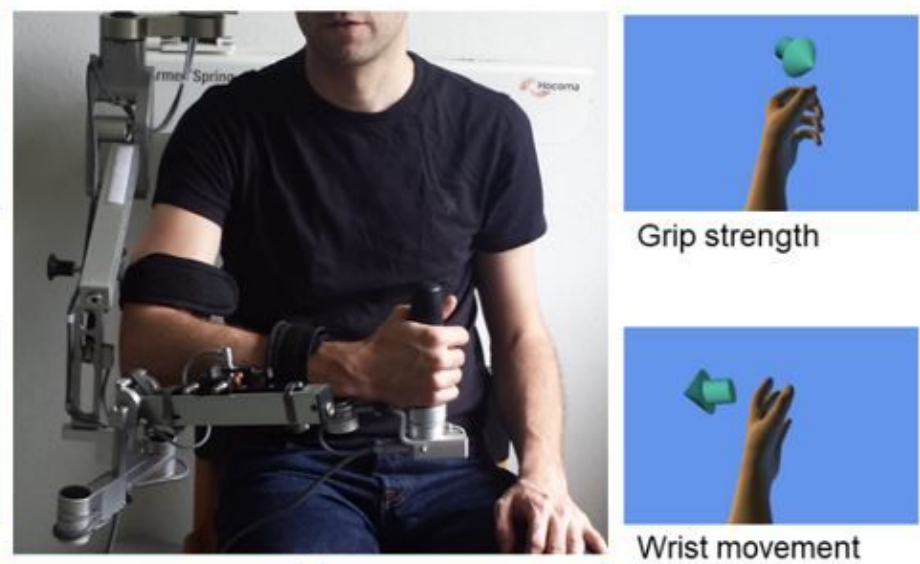

Grip strength
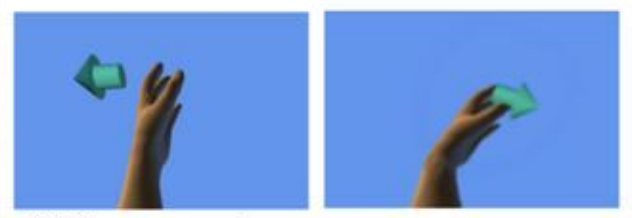

Wrist movement
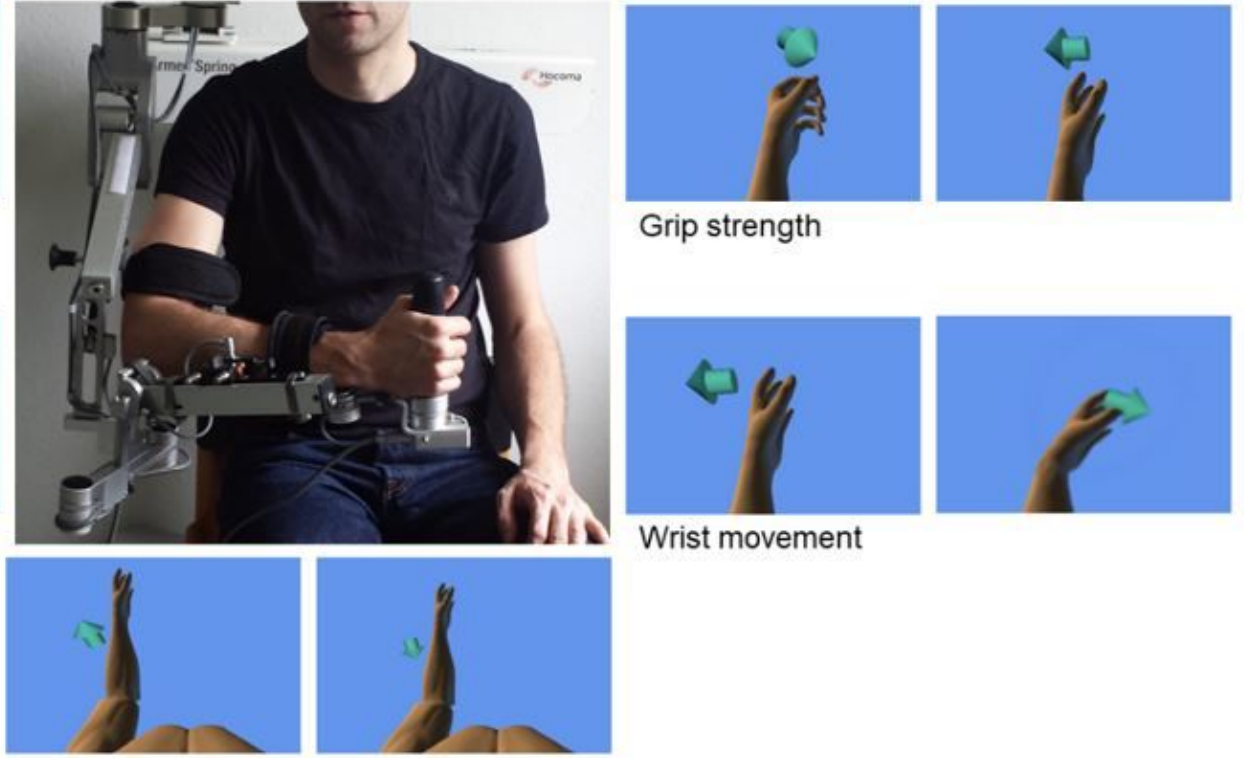

Elbow movement

\section{Figure 1}

Subject with exoskeleton (in the center); 3D visualization of the instructed movements of the five assessment tasks. 


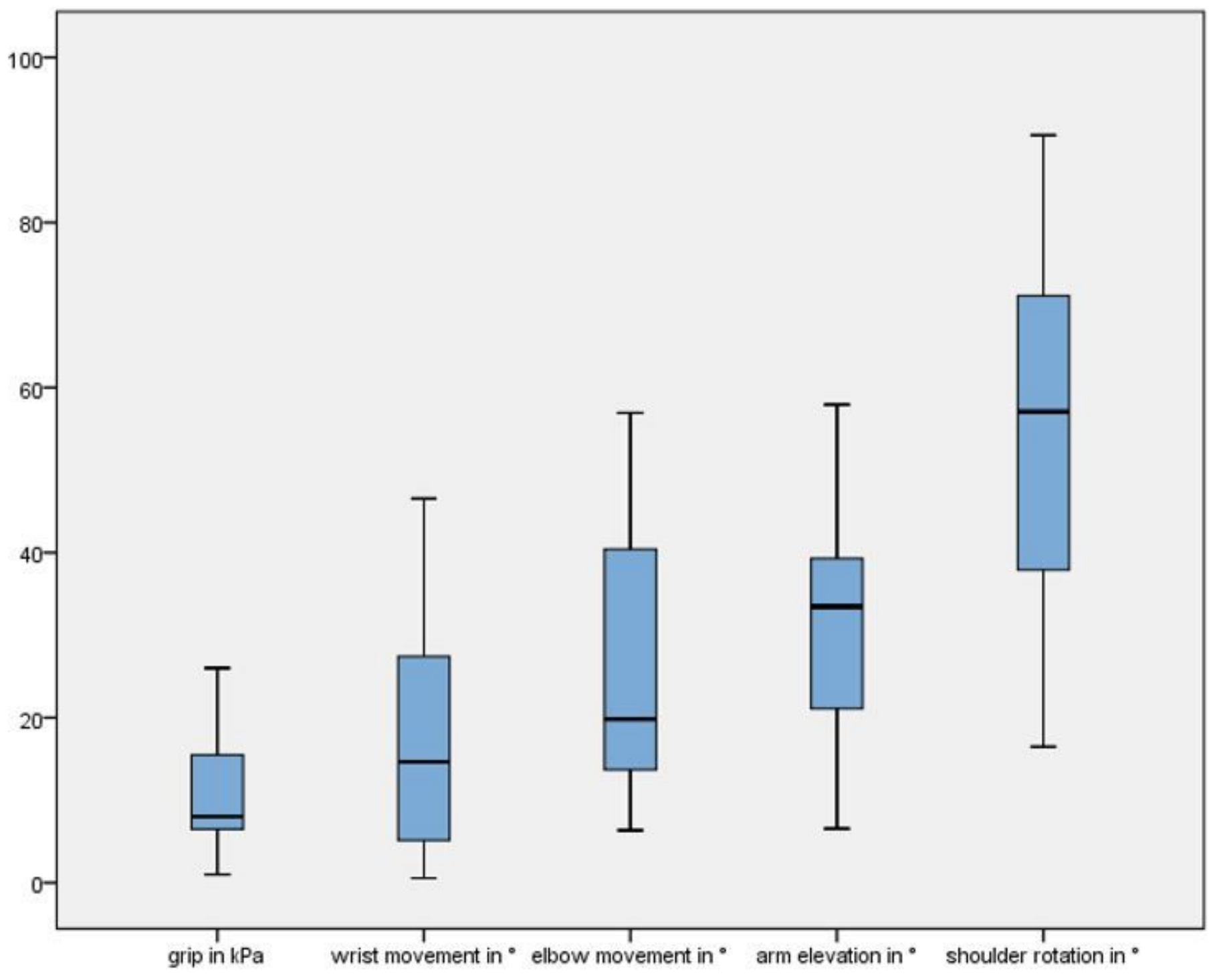

Figure 2

Boxplots of kinematic assessment results of all patients 

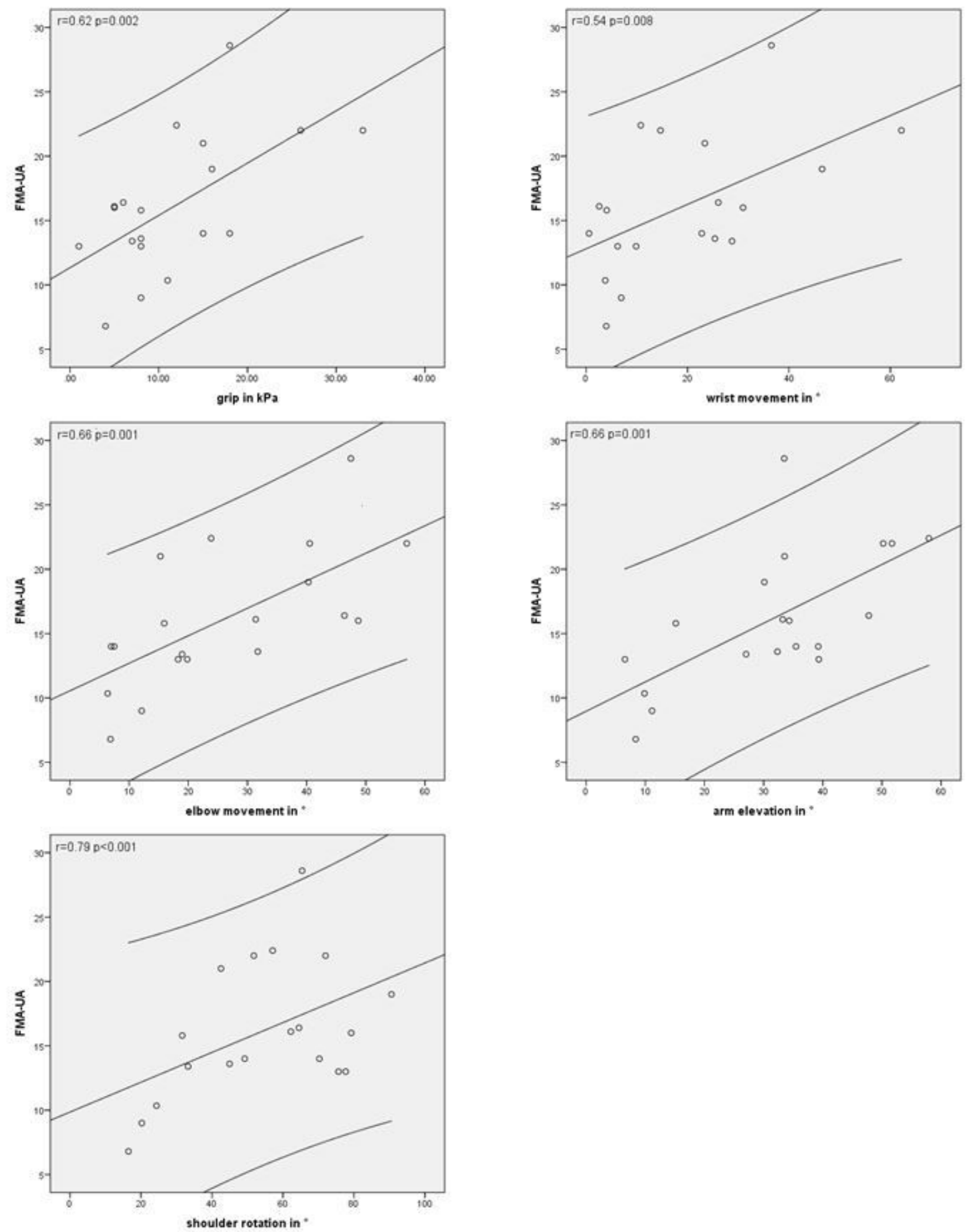

\section{Figure 3}

Scatter plots with Pearson correlation between clinical (UE-FMA scale) and kinematic parameters (grip force, wrist/elbow/arm/shoulder movement) 

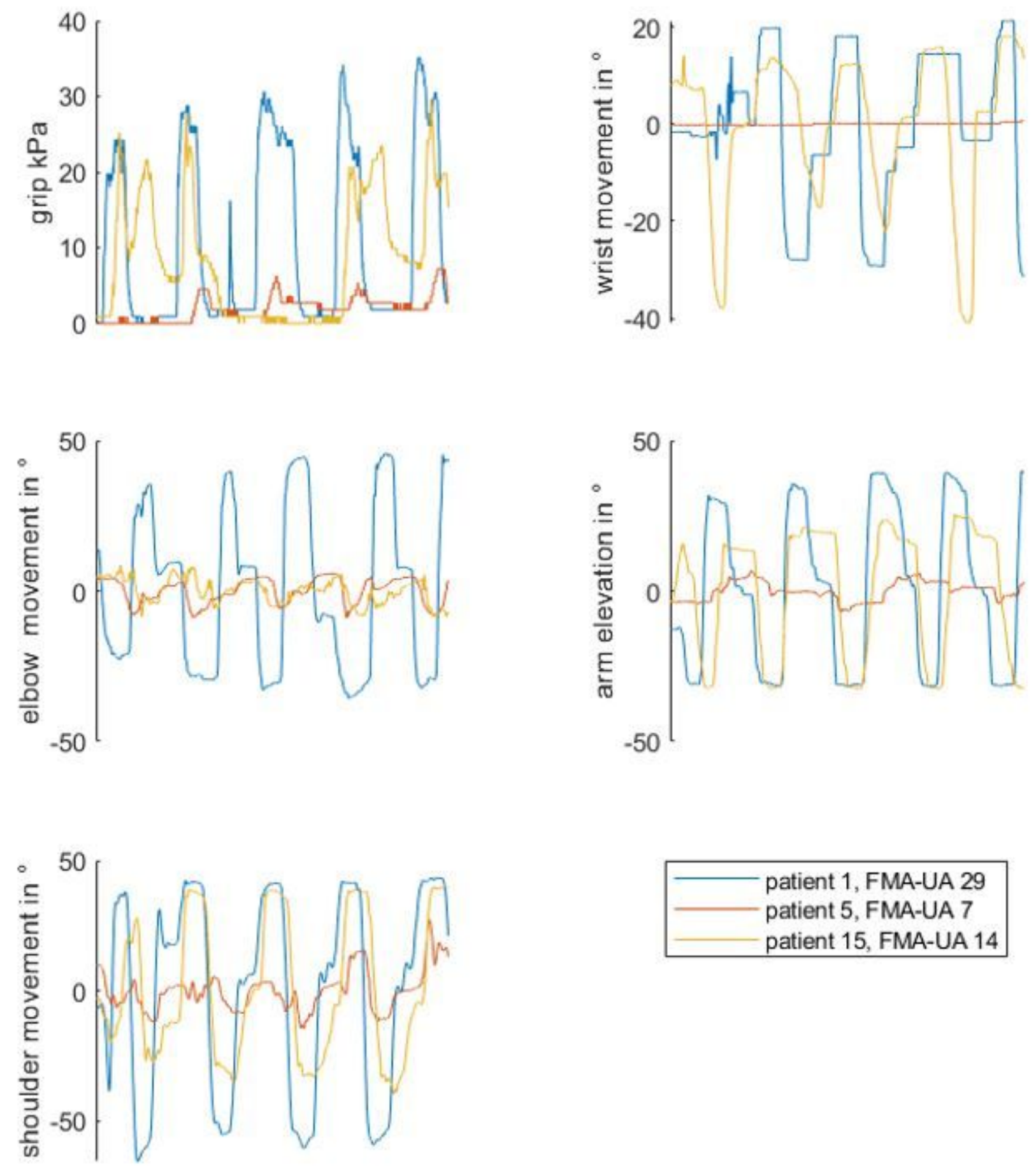

Figure 4

Exemplary real-time kinematic data of patients 1, 5 and 15 of the five tasks. 